\title{
A longitudinal study of the cardiac response during a problem-solving task and its relationship to general cognitive function
}

\author{
WILLIAM G. STEELE AND MICHAEL LEWIS, THE FELS \\ RESEARCH INSTITUTE, Yellow Springs, Ohio
}

It has been hypothesized that cardiac responsivity should vary as a function of the $S$ 's intended transaction with his environment. $A$ developmental inquiry was instigated to determine the cardiac response to a mental arithmetic problem. Across age levels, Ss showed cardiac acceleration during problem solution. While there was no developmental pattern in the amount of acceleration, the results did indicate that the amount of cardiac acceleration was positively related to $S s^{\prime} I Q$.

The relationship between cardiac responsivity and various aspects of intellectual functioning has been the subject of increased interest. An integrative theory has been presented by Lacey (Lacey, Kagan, Lacey, \& Moss, 1963; Lacey, 1967) in which cardiac responsivity is related to the S's intended transaction with his environment. According to this theory, cardiac deceleration accompanies mental incorporation of environmental stimuli, and cardiac acceleration accompanies rejection of the environment or mental elaboration, as during the solution of a problem. This formulation has been supported by findings of several investigators using both adult Ss (Blatt, 1961; Obrist, 1963; Coquery \& Lacey, 1966; Steele \& Koons, 1968) and infants (Lewis, Bartels, \& Goldberg, 1967; Lewis \& Goldberg, 1967; Chase \& Graham, 1967). Lacey et al (1963) further suggest that in addition to the nature of the specific task, the degree of cardiac involvement should be related to the $S$ 's general intellectual function. In this latter study, data were presented to indicate that verbal IQ was related to cardiac reactivity in a variety of stimulus situations.

While there has been much investigation of cardiac response to a variety of $S$ behaviors, there has been little developmental inquiry into the cardiac concomitant of problem-solving. The present study, therefore, seeks to investigate the relationship between general cognitive function and cardiac response over a wide age span.

Method. The data reported here are derived from a larger investigation being conducted and are presented here as a preliminary report of our findings. While their cardiac function was monitored, a total of $150 \mathrm{Ss}$ ranging in age from six to 27 years for whom IQ data were available were given a mental arithmetic task.

For analysis, Ss were divided into four age groups: Group I, 26 Ss, 6-8 years old; Group II, 34 Ss, 9-11 years old; Group III, 29 Ss, 12-15 years old; and Group IV, 61 Ss, 16-27 years old.

The ECG signals were received by means of Beckman silver silver-chloride electrodes. The ECG signal was first amplified through a Tecktronix FM 122 pre-amplifier and then fed to the driver amplifier of a Grass Model 7 polygraph. The ECG and stimulus condition were recorded on a punch paper tape system (Welford, 1962).

The Ss were monitored in the equipment room by means of a closed circuit television. The arithmetic problems were printed singly on blank $3 \times 5$ in. cards which were shown one at a time to the $S$ by an $E$. The mental arithmetic task consisted of five simple problems which were administered in order of increasing difficulty. There was a short break between each problem. This constituted the base period. Coding was controlled from a small panel by $E$ and was recorded on the polygraph and punch paper systems.

The $S$ was seated in a comfortable armchair, and a pair of electrodes were attached to $S$ for recording the electrocardiogram. The mental arithmetic task was then explained to $\mathrm{S}$. It consisted of solving addition problems mentally and announcing the answers aloud. Three practice trials were given to insure that each $S$ understood the task. Typical of the problems administered were the following: $9+2+5=?, 11+10=?, 18+81=?, 41+59=?, 25+37=$ ?. A 10-min rest period was presented prior to the problem-solving period in order to obtain basic resting HR data.
Results. A median curve for the five problems was obtained for each S. Figure 1 presents the median cardiac response curve for each of the four age groups during the performance of the mental arithmetic task. The $X$ axis represents the number of cardiac cycles or R-R intervals from onset of the task, while the $Y$ axis represents the heart rate for each cardiac cycle. One cycle prior to onset and seven cycles after onset of the task are presented, and represent in actual time approximately $1 \mathrm{sec}$ prior to onset and $5 \mathrm{sec}$ of task solution. Because of the variable time lengths of solution, $5 \mathrm{sec}$ represents the response time for problem solution for $50 \%$ or more of Ss. Therefore, the first $5 \mathrm{sec}$ or seven cardiac cycles were set as the criterion for observing the cardiac response to the task.

As would be expected, there is a perfect age ordering of the base heart rate, the youngest group having the highest resting rate. A Kruskal-Wallis analysis of variance indicates significant age differences $(H=12.13, \mathrm{df}=3, \mathrm{p}<.02)$. Data from Fig. 1 indicate that for all age groups, the cardiac concomitant of problem-solving is an immediate cardiac acceleration lasting for approximately three cardiac cycles, followed by a return to or below the resting level.

In order to determine whether the acceleration following task onset was significant, analyses for each age level were performed. In the first two analyses, the cardiac beat cycle prior to onset of the task was compared to the first and second cardiac cycle during task solution $(\mathrm{p}<.001$ for each analysis by Wilcoxon Paired

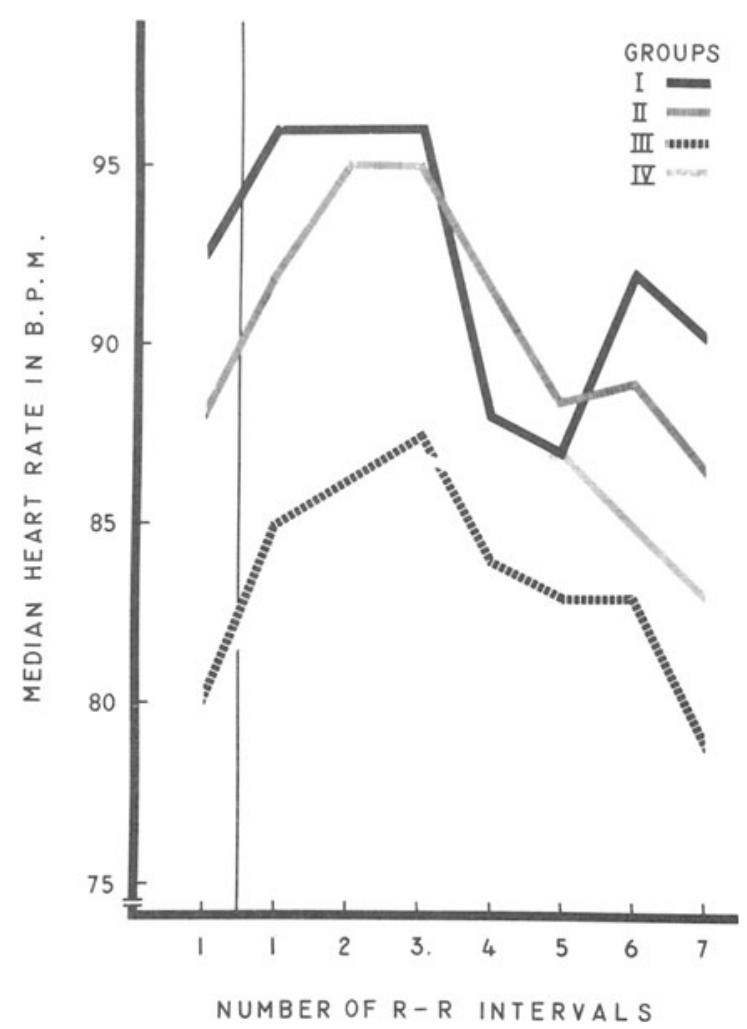

Fig. 1. Cardiac response by age to the presentation of a mental arithmetic task. The $X$ axis represents the number of cardiac cycles from the onset of the problem until problem solution, while the $Y$ axis represents the heart rate associated with each cycle. 
Replicates Test). On the third analysis, the cardiac cycle prior to onset of the task was compared to the fastest cycle during task solution, and this result was also highly significant $(p<.001)$. The results, therefore, indicate an immediate and significant cardiac response of acceleration during the mental arithmetic task.

To determine whether there are any age differences in the degree of acceleration, it was necessary to control the differential base levels. An autonomic lability score (ALS) proposed by Lacey (1956) was utilized. This regression procedure controlled for the different levels and when age differences were compared using this technique, the results failed to indicate any significant age differences in acceleration during problem solution.

It was predicted that general intellectual function should be related to the degree of cardiac responsivity, in this case, cardiac acceleration. To test this prediction, standard WISC and WAIS full-scale intelligence test scores were obtained for each S. For each age level, the IQ score was compared to a measure of cardiac acceleration (base minus fastest cardiac cycle). Across all ages there was a low positive correlation such that the greater the acceleration, the higher the IQ $(r=.18, \mathrm{df}=149, \mathrm{p}<.05)$. A comparison by age group revealed that while this relationship held across all groups, it was significant only for the 6- to 8-year-olds ( $r$ $=.34, \mathrm{df}=26, \mathrm{p}<.05$ ).

Discussion. One reason for the low positive correlation may rest on the fact that the mental arithmetic problem was too simple for the majority of the Ss. This hypothesis gains support from the fact that it was the youngest group, presumably those who would find the problems most difficult, who showed the highest correlation. Thus, while there was a significant cardiac acceleration, there was not ample task difficulty to differentiate this acceleration by levels of IQ.

In general, during the solution of a simple mental arithmetic problem, Ss in all age levels showed an initial cardiac acceleration followed by a return to base level or a slight deceleration. Because most Ss solved the problems very quickly (within $5 \mathrm{sec}$ ), only the first seven cardiac cycles were observed. The nature of the response after the initial seven cycles is not available, but the results do support the belief that cardiac acceleration accompanies the solution of a mental arithmetic problem.

The degree of cardiac acceleration did not vary as a function of age and indicates that the cardiac response concomitant of problem-solving is consistent over a wide age span. However, this statement must be tempered to take into account the ease of this particular task. Thus, it is possible that age differentiation, like 10 , would not be observed when the mental elaboration was not difficult enough.
These data, as well as others, suggest that cardiac responsivity is influenced by at least three factors. The first is the intent of the $S$, that is, the $S$ 's desire to note and detect environmental stimuli or to engage in mental elaboration. Second, the state of the $S$, i.e., his capacity in terms of IQ and such motivational variables as high achievement needs. And third, the objective environmental situation, i.e., the difficulty of the task.

\section{REFERENCES}

BLATT, S. J. Patterns of cardiac arousal during complex mental activity. $J$. abnorm soc. Psychol, 1961, 63, 272-282.

COQUERY, J., \& LACEY, J. I. The effect of foreperiod duration on the components of the cardiac response during the foreperiod of a reactiontime experiment. Paper presented at the Sixth Annual Meeting of the Society for Psychophysiological Research, Denver, October, 1966.

CHASE, W. G., \& GRAHAM, F. K. Heart rate response to non-signal tones. Psychon. Sci, 1967, 9, 181-182.

LACEY, J. l. Somatic response patterning and stress: Some revisions of the activation theory. In R. Trumbull (Ed.), Psychological stress: Issue in research. New York: Appleton-Century-Crofts, 1967.

LACEY, J. I. The evaluation of autonomic responses: Toward a general solution. Ann. N. Y. Acad. Sci, 1956, 67, 123-164.

LACEY, J. I., KAGAN, J., LACEY, B. C., \& MOSS, H. A. The visceral level: Situational determinants and behavioral correlates of autonomic response patterns. In P. H. Knapp (Ed.), Expressions of the emotions in man. New York: International Universities Press, 1963.

LEWIS, M., BARTELS, B., \& GOLDBERG, S. State as a determinant of infants' heart rate response to stimulation. Science, $1967,155,486-488$.

LEWIS, M., \& GOLDBERG, S. Habituation differences to tactile stimulation for waking and sleeping infants. Paper presented at the Seventh Annual Meeting of the Society for Psychophysiological Research, San Diego, October, 1967.

OBRIST, P. Cardiovascular differentiation of sensory stimuli. Psychosom. Med., 1963, 25, 450-459.

STEELE, W. G., \& KOONS, P. B., Jr. Cardiac response to mental arithmetic under quiet and white noise distraction. Psychon. Sci., 1968, 11, 273-274.

WELFORD, N. T. The SETAR and its uses for recording physiological and behavioral data. IRE trans. biomed. Elect., 1962 (9), 185-189.

\section{NOTE}

1. This research was supported in part by Grants No. HD-00868, FR-00222 and FR-05537 from the National Institute of Mental Health, United States Public Health Service. The authors wish to thank Joan King for her assistance in data analysis. 